\title{
A boundary integral equation method for the two-dimensional diffusion equation subject to a non-local condition
}

\author{
W.T. Ang* \\ Computational and Mathematical Sciences Group, Faculty of Information Technology, Universiti Malaysia Sarawak, 94300 Kota Samarahan, Malaysia
}

Received 24 January 2000; revised 26 October 2000; accepted 26 October 2000

\begin{abstract}
A boundary integral equation method is proposed for the numerical solution of the two-dimensional diffusion equation subject to a nonlocal condition. The non-local condition is in the form of a double integral giving the specification of mass in a region which is a subset of the solution domain. A specific test problem is solved using the method. (C) 2001 Elsevier Science Ltd. All rights reserved.
\end{abstract}

Keywords: Boundary element method; Diffusion equation; Laplace transform; Non-local condition

\section{Introduction}

In non-dimensionalized form, the partial differential equation that governs two-dimensional linear and isotropic diffusion processes is given by

$$
\frac{\partial^{2} u}{\partial x^{2}}+\frac{\partial^{2} u}{\partial y^{2}}=\frac{\partial u}{\partial t} \text {. }
$$

A class of problems of practical interest is to solve Eq. (1) for the unknown function $u(x, y, t)$ for time $t \geq 0$ in a twodimensional region $R$ (on the $0 x y$ plane) subject to the initial and boundary conditions

$$
\begin{aligned}
& u(x, y, 0)=f(x, y) \quad \text { for }(x, y) \in R, \\
& u(x, y, t)=g(x, y, t) \quad \text { for }(x, y) \in C_{1} \text { and } t \geq 0, \\
& u(x, y, t)=h(x, y) z(t) \quad \text { for }(x, y) \in C_{2} \text { and } t \geq 0, \\
& \frac{\partial}{\partial n}[u(x, y, t)]=k(x, y, t) \quad \text { for }(x, y) \in C_{3} \text { and } t \geq 0,
\end{aligned}
$$

and the non-local (integral) condition

$$
\iint_{S} u(x, y, t) \mathrm{d} x \mathrm{~d} y=m(t) \quad \text { for } t \geq 0 .
$$

where $f, g, h, k$ and $m$ are known and suitably prescribed functions, $z$ is an unknown function to be determined, the region $R$ is bounded by a simple closed curve $C$, the curves

\footnotetext{
* Fax: +60-82-672301.

E-mail address: wtang@mailhost.fit.unimas.my (W.T. Ang).
}

$C_{1}, C_{2}$ and $C_{3}$ are non-intersecting and such that $C_{1} \cup C_{2} \cup$ $C_{3}=C, S$ is a given subregion of $R$ that is independent of time $t$ and is bounded by a simple closed curve $D$ given by $D=C_{2} \cup C_{4}$, the open curve $C_{4}$ lies completely in the interior of $R$, and $\partial u / \partial n=\mathbf{n} \cdot \nabla u, \mathbf{n}$ is the unit normal vector on $C$ pointing away from $R$. From a physical standpoint, Eq. (6) specifies the total amount of mass of the diffusing quantity $u$ (or the total amount of heat energy, in the case of heat diffusion) which the region $S$ can possess at any time $t$. Condition (4) with $z(t)$ being unknown implies that the concentration of the diffusing quantity (or the temperature) on some part of the boundary must be controlled in a certain way in order that the region $S$ carries the specified amount of mass (or heat energy). For a sketch of the geometry of the problem, refer to Fig. 1.

The class of problems defined by Eqs. (1)-(6) arises in many practical applications in heat transfer, control theory, thermoelasticity and medical sciences. A specific application which involves the use of the absorption of light to measure the concentration of a diffusing chemical is described by Noye and Dehghan [6]. Special cases of the problem, such as $R$ being a rectangular region, have been solved directly by many researchers, e.g. Gumel et al. [5], Noye and Dehghan [6,7], Noye et al. [8], Cannon et al. [3], and Wang and Lin [11] using the finite-difference methods. With the exception of Ref. [6], the case $S=R$, i.e. $C_{4}=$ $C_{1} \cup C_{3}$, was studied in all the references just cited.

The present paper makes use of a boundary integral equation method (BIEM) for the numerical solution of Eqs. (1)-(6) in the Laplace transform (LT) space. The physical solution is recovered by using the Stehfest 